\title{
Lower bound on fermion binding energies
}

\author{
Olivier Juillet, ${ }^{1}$ Sonia Fleck, ${ }^{1}$ Lukas Theussl, ${ }^{2}$ Jean-Marc Richard, ${ }^{2}$ and Kálmán Varga ${ }^{3}$ \\ ${ }^{1}$ Institut de Physique Nucléaire, Université Claude Bernard, CNRS-IN2P3, 43 boulevard du 11 Novembre 1918, \\ F 69622 Villeurbanne, France \\ ${ }^{2}$ Institut des Sciences Nucléaires, Université Joseph Fourier, CNRS-IN2P3, 53 avenue des Martyrs, F 38026 Grenoble, France \\ ${ }^{3}$ Solid State Division, Oak Ridge National Laboratory, Oak Ridge, Tennessee 37381 \\ and Institute for Nuclear Research of the Hungarian Academy of Sciences, Debrecen, Hungary
}

(Received 10 August 2000; published 22 January 2001)

\begin{abstract}
We derive a lower bound for the ground-state energy $E^{F}(N, S)$ of $N$ fermions with total spin $S$ in terms of binding energies $E^{F}(N-1, S \pm 1 / 2)$ of $(N-1)$ fermions. Numerical examples are provided for some simple short-range or confining potentials.
\end{abstract}

DOI: 10.1103/PhysRevB.63.073102

PACS number(s): 05.30.Fk, 21.45.+v

There is a persisting interest in deriving lower bounds on the binding energy of $N$-particle systems. Some early investigations were motivated by fundamental studies on the thermodynamic limit or the stability of matter. ${ }^{1}$ Also, the search for a lower bound is rather natural once an upper bound is provided by variational estimates.

Many efforts have been devoted in particular to express a bound on the binding energy $E_{N}$ of an $N$-body system in terms of an $(N-1)$-body energy $E_{N-1}$ with modified constituent mass or interaction strength. Thanks to a proper account for the center-of-mass motion of the subsystems, ${ }^{2}$ the situation is now rather satisfactory in the zero-spin boson sector: the bound of Hall and $\mathrm{Post}^{2}$ is saturated for the harmonic oscillator and approaches closely the exact result for many other potentials. An extension has been derived for three and four particles with different masses $^{3}$ where, again, saturation is obtained in the case of harmonic forces.

The situation is far more difficult for fermions. The elegant bound derived by Lévy-Leblond ${ }^{1}$ suffers from the fact that the energy of $(N-1)$-body subsystems is replaced by its rest energy without including its overall kinetic energy. As a result, saturation is never reached.

A significant but partial improvement was obtained by Basdevant and Martin, ${ }^{4}$ who used subtle convexity inequalities. Their bound improves that of Lévy-Leblond in some cases, and becomes exact for the harmonic oscillator. Their approach, however, is restricted to confining potentials $r^{q}$, $q \geqslant 1$, or superpositions of such power-law potentials with positive weight factors.

In this Brief Report, we use group-theoretical techniques to derive a general lower bound on the $N$-body energy of an interacting system of particles with internal degrees of freedom. The decomposition of the Hamiltonian is supplemented by considerations on the symmetry structure of the wave function. Numerical tests are presented and possible generalizations are sketched, in particular for quantum dots, i.e., particles interacting both among themselves and with an external potential.

Let us consider first $N$ identical particles of mass $m$ whose interaction does not depend on their spins. This corresponds to the Hamiltonian

$$
H_{N}(m, g)=\sum_{i} \frac{\mathbf{p}_{i}^{2}}{2 m}+g \sum_{i<j} V\left(r_{i j}\right),
$$

where $r_{i j}=\left|\mathbf{r}_{j}-\mathbf{r}_{i}\right|$ is the distance between two particles. The case of particles with different masses is treated in Ref. 3.

A first decomposition of this Hamiltonian is ${ }^{1,5}$

$$
H_{N}(m, g)=\frac{1}{N-2} \sum_{i} H_{N-1}^{(i)}\left(\frac{N-1}{N-2} m, g\right),
$$

where the constituent mass in $H_{N-1}$ is increased, since the kinetic energy $\mathbf{p}_{1}^{2} /(2 m)$, for instance, is shared by $(N-1)$ terms. The $i$ th particle is absent from $H_{N-1}^{(i)}$. Using the variational principle with the ground state of the $\mathrm{N}$-body system as a trial wave function leads to an inequality on the ground-state energies $E_{N},{ }^{1,5}$

$$
E_{N}(m, g) \geqslant \frac{N}{N-2} E_{N-1}\left(\frac{N-1}{N-2} m, g\right),
$$

which can be rewritten differently, using the obvious identity $E(\alpha m, g)=E(m, \alpha g) / \alpha$. This inequality is never saturated, because the overall translation energy of the $(N-1)$-body subsystems within the $N$-body system is neglected. An improvement consists in replacing the decomposition (2) by the identity

$$
\widetilde{H}_{N}(m, g)=\frac{1}{N-2} \sum_{i} \widetilde{H}_{N-1}^{(i)}\left(\frac{N}{N-1} m, g\right),
$$

relating the translation-invariant Hamiltonians

$$
\tilde{H}_{N}=H_{N}-\frac{\left(\sum \mathbf{p}_{i}\right)^{2}}{2 N m} .
$$

This leads to the new inequality

$$
E_{N}(m, g) \geqslant \frac{N}{N-2} E_{N-1}\left(\frac{N}{N-1} m, g\right) .
$$

As $N m /(N-1)$ is smaller than $(N-1) m /(N-2)$ for $N$ $\geqslant 3$, the bound (6) is better than (3), since any binding energy in a given potential is a decreasing function of the constituent mass.

By recursion, an inequality such as (3) or (6) provides a bound on $E_{N}$ in terms of the two-body energy $E_{2}$. In the 
TABLE I. Results for Yukawa $(Y)$, Gaussian $(G)$, and exponential $(E)$ potentials. The range parameter is set to unity by rescaling. The quantity shown is the ratio of the computed energy to the bound. In the first line, the entries are the number of particles, the boson $(B)$ or fermion $(F)$ character, and the total spin. The fourbody energy is compared to the three-body one, as per Eq. (3) for bosons and Eq. (12) for fermions. The constituent mass is set to $m=1$ for $N=3$ particles and $m=3 / 4$ for $N=4$.

\begin{tabular}{rrrccccc}
\hline \hline & $g$ & $3, B, 0$ & $3, F, 1 / 2$ & $3, F, 3 / 2$ & $4, B, 0$ & $4, F, 0$ & $4, F, 2$ \\
\hline$Y$ & 8 & 0.933 & 0.673 & 0.759 & 0.966 & 0.743 & 0.855 \\
& 15 & 0.943 & 0.757 & 0.930 & 0.971 & 0.806 & 0.964 \\
\hline \multirow{2}{*}{$G$} & 10 & 0.996 & 0.960 & 0.887 & 0.998 & 0.792 & 0.945 \\
& 20 & 0.999 & 0.995 & 0.994 & 0.999 & 0.898 & 0.997 \\
\hline \multirow{2}{*}{$E$} & 6 & 0.988 & 0.906 & 0.843 & 0.994 & 0.795 & 0.913 \\
& 12 & 0.994 & 0.974 & 0.982 & 0.997 & 0.886 & 0.991 \\
\hline \hline
\end{tabular}

case of the harmonic oscillator for bosons, the bound derived from (6) is saturated, whereas the bound derived from (3) is smaller by a factor $\sqrt{2}$ than the exact result at large $N$.

Some numerical illustration of the inequality (6) for bosons is provided in Table I. Other examples are given in Ref. 2.

In the case of fermions, the bound (6) is not satisfactory. Indeed, it is not necessarily the ground state of $\widetilde{H}_{N-1}$ that is relevant, but the lowest state with quantum numbers compatible with the symmetry of the $N$-body state of interest. Thus looking at the structure of the wave function seems important to improve the inequalities.

Since our Hamiltonian acts in the orbital Hilbert space only, the behavior of the wave function with respect to the space variables has to be specified by an irreducible representation of the symmetry group $S_{N}$. This corresponds to a partition $[\lambda]=\left[\lambda_{1}, \lambda_{2}, \ldots, \lambda_{n}\right]$ of the $N$ particles defining an invariant orbital subspace spanned by orthogonal states that are associated to a Young tableau or a Yamanouchi symbol $r .{ }^{6}$ Both specify the transformation under permutation, and the matrix element of any transposition operator $P_{i j}$ is known analytically. For example, in the case of three particles, we can have the following partitions:

$$
[3], \quad[2,1], \quad[1,1,1] \text {. }
$$

The first and the third ones are in the familiar symmetric and antisymmetric representations of dimension 1, respectively. The second one, of dimension 2 , is the mixed symmetry representation spanned by the two states $r=(2,1,1)$ and $r$ $=(1,2,1)$, which are, respectively, symmetric and antisymmetric in the exchange of particles 1 and 2 .

Each orbital state $|N,[\lambda], r\rangle_{o}$ must be associated with a similar state $|N,[\bar{\lambda}], \bar{r}\rangle_{i}$ for the intrinsic degrees of freedom such as spin, isospin, color, etc. The coupling of $[\bar{\lambda}]$ to $[\lambda]$ gives a symmetric representation $[N]$ of $S_{N}$ for bosons or a fully antisymmetric representation $\left[1^{N}\right]$ for fermions. This implies that $[\bar{\lambda}], \bar{r}$ are identical to $[\lambda], r$ when we are dealing with bosons, while for fermions, $[\bar{\lambda}], \bar{r}$ correspond to a Young tableau with rows and columns interchanged. ${ }^{6}$

The $S_{N}$ Clebsch-Gordan coefficients $\langle[\lambda] r[\lambda] r \mid[N]\rangle$ and $\left\langle[\lambda] r[\bar{\lambda}] \bar{r} \mid\left[1^{N}\right]\right\rangle$ are known explicitly, ${ }^{6}$ and the $N$-body state reads ( $\epsilon=1$ for bosons, -1 for fermions)

$$
|N,[\lambda]\rangle_{\epsilon}=d_{[\lambda]}^{-1 / 2} \sum_{r} \epsilon^{\sigma}|N,[\lambda], r\rangle_{o}|N,[\bar{\lambda}], \bar{r}\rangle_{i}
$$

where

$$
d_{[\lambda]}=N ! \frac{\prod_{1 \leqslant i<j \leqslant n}\left(\lambda_{i}-\lambda_{j}+j-i\right)}{\prod_{1 \leqslant i \leqslant n}\left(\lambda_{i}+n-i\right) !}
$$

is the dimension of the representation $[\lambda]$ and $\sigma$ is the number of permutations that have to be performed to obtain the Yamanouchi symbol from the normal one. ${ }^{6}$ To obtain the $(N-1)$-body parts of state (8), one has now just to remove the $N$ th particle in each symbol $r$, thus leading to an $(N$ $-1)$-Yamanouchi symbol that belongs to a different orbital symmetry $\quad[\lambda]_{p}=\left[\lambda_{1}, \ldots, \lambda_{p-1}, \lambda_{p}-1, \lambda_{p+1}, \ldots, \lambda_{n}\right]$, where $p$ is the row number of particle $N$ in the original Young tableau. Moreover, this new symbol needs $a$ $=\sum_{q>p=1}^{N} \lambda_{q}$ fewer permutations than $r$ to appear in the normal form. Hence the $N$-body wave function (8) can be rewritten as

$$
\sum_{p} \frac{d_{[\lambda]_{p}}^{1 / 2}}{d_{[\lambda]}^{1 / 2}} \epsilon^{a}\left|\left(N-1,[\lambda]_{p}\right)_{\epsilon}(1,[1])\right\rangle_{[\lambda], \epsilon},
$$

with $p$ running over all the lines of $[\lambda]$ where a box can be dropped. When this is used with the decomposition (4) of the Hamiltonian, it leads to a new and very general inequality

$$
E_{N}^{[\lambda]}(m, g) \geqslant \frac{N}{N-2} \sum_{p} \frac{d_{[\lambda]_{p}}}{d_{[\lambda]}} E_{N-1}^{[\lambda]_{p}}\left(\frac{N}{N-1} m, g\right) .
$$

If one considers, as a first application, a system of spinless bosons for which the orbital Young pattern $[\lambda]$ is necessarily symmetric, the inequality (11) coincides with our previous result (6).

Consider now fermions with spin $1 / 2$. The conjugate partition $[\bar{\lambda}]$ of $[\lambda]$ is an irreducible representation of $U(2)$ and contains at most two rows that are related to the spin $S$ by the relations $\bar{\lambda}_{1}+\bar{\lambda}_{2}=N$ and $\bar{\lambda}_{1}-\bar{\lambda}_{2}=2 S$. Writing our inequality (11) with $[\bar{\lambda}]$ gives the following result: the ground-state energy of $N$ spin 1/2 fermions with total spin $S$ is bound in terms of binding energies of $(N-1)$-body systems with neighboring spins $S \pm 1 / 2$, 


$$
\begin{aligned}
E_{N}^{S}(m, g) \geqslant & \frac{1}{(N-2)(2 S+1)} \\
& \times\left[S(N+2 S+2) E_{N-1}^{S-1 / 2}\left(\frac{N m}{N-1}, g\right)\right. \\
& \left.+(S+1)(N-2 S) E_{N-1}^{S+1 / 2}\left(\frac{N m}{N-1}, g\right)\right] .
\end{aligned}
$$

The generalization is straightforward: the energetically favored state has the most symmetric space partition $[\lambda]$ that admits a conjugate partition $[\bar{\lambda}]$ which can be a representation of $U(\Omega)$, where $\Omega$ is the number of intrinsic degrees of freedom. As a result, $[\bar{\lambda}]$ has a maximum of $\Omega$ rows and $[\lambda]=\left[\Omega^{\nu}, N-\nu \Omega\right]$ for the $N$-body ground state with $\nu$ the integer part of $N / \Omega$. Denoting by $E_{N-1}^{0}$ the binding energy of $(N-1)$ particles with the favored orbital symmetry $\left[\Omega^{\nu}, N-\nu \Omega-1\right]$ and by $E_{N-1}^{1}$ the energy associated to the first excited partition $[\lambda]=\left[\Omega^{\nu-1}, \Omega-1, N-\nu \Omega\right]$, the relation (11) takes the form

$$
\begin{aligned}
E_{N}(m, g) \geqslant & \frac{1}{(N-2)(1+\Omega+\nu \Omega-N)} \\
& \times\left[(N-\nu \Omega)(1+\Omega+\nu+\nu \Omega-N) E_{N-1}^{0}\right. \\
& \times\left(\frac{N m}{N-1}, g\right)+\nu(\Omega+1)(\Omega+\nu \Omega-N) E_{N-1}^{1} \\
& \left.\times\left(\frac{N m}{N-1}, g\right)\right]
\end{aligned}
$$

We have calculated the lower bound and the exact energy with a selection of potentials, using the method of Gaussian expansion described, for instance, in Ref. 7. The variational parameters are determined by a stochastic optimization. ${ }^{8}$ The method has proved to be powerful and reliable.

In Table I, we show the ratio of the accurately computed energy to the lower bound for monotonic short-range potentials of Yukawa, $V=-g \exp (-r) / r ;$ Gaussian, $V$ $=-g \exp \left(-r^{2}\right)$, and exponential, $V=-g \exp (-r)$ types. For $N=3$ and $S=1 / 2$, each pair is in an equal-weight admixture of singlet and triplet, while $S=3 / 2$ involves only triplet states. For $N=4$ and $S=0$ (2), each three-body subsystem has $S=1 / 2(3 / 2)$.

It appears from Table I that the bound is close to the exact result, especially for antisymmetric orbital wave functions ( $N=3, S=3 / 2$ or $N=4, S=2$ ) and for deeply bound states.

The results in Fig. 1 correspond to power-law potentials $r_{i j}^{q}$ with $q \geqslant 1$. For comparison, we also display the bound by Lévy-Leblond, ${ }^{1}$ initially designed for large systems but also applicable at small $N$. The decomposition

$$
H_{N}(m, g)=\frac{1}{2} \sum_{i} \sum_{j \neq i}\left[\frac{\mathbf{p}_{j}^{2}}{(N-1) m}+g V\left(r_{j i}\right)\right]
$$

expresses $H_{N}$ in terms of Hamiltonians with $(N-1)$ independent particles. For fermions, one gets

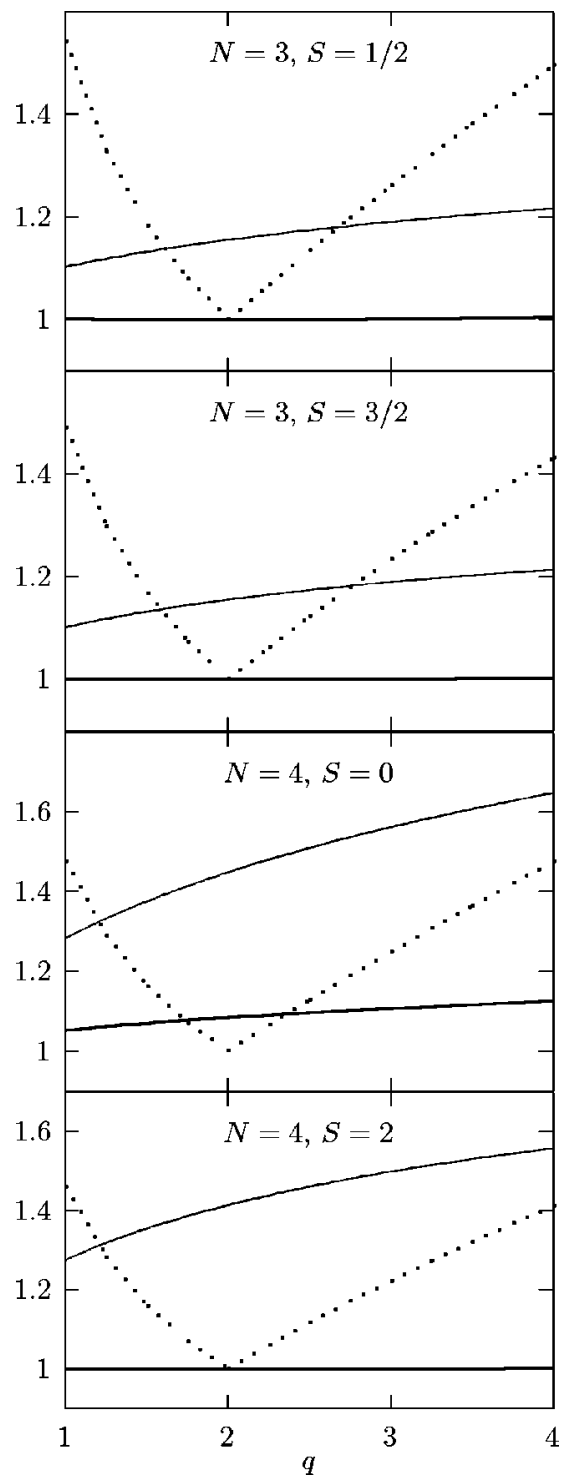

FIG. 1. Comparison of various lower bounds for $N=3$ or 4 fermions with total spin $S$, in the case of power-law potentials $r^{q}$. The quantity shown is the ratio of the exact energy to our bound (thick line) to that of Basdevant and Martin (dotted line) and that of Lévy-Leblond (thin line).

$$
E_{N}(m, g) \geqslant \frac{N}{2} f_{N-1}(m(N-1), g),
$$

where $f_{N}$ is the cumulated energy of a system of $N$ independent fermions, a notation borrowed from Ref. 4. In Eq. (15), the translation energy of the $(N-1)$-body subsystem is neglected. This problem was already raised by Manning and Balbutsev in their discussion of the work of Carr and Post. ${ }^{9}$ These authors have proposed several improved bounds expressed like Eq. (15) in terms of $(N-1)$ independent particles.

Also shown in Fig. 1 is the bound of Basdevant and Martin, ${ }^{4}$ who started from the identity

$$
\sum_{i} \mathbf{p}_{i}^{2}+\mathbf{r}_{i}^{2}=\frac{\mathbf{P}^{2}}{N}+N \mathbf{R}^{2}+T_{\mathrm{r}}+\frac{1}{N} \sum_{i<j} r_{i j}^{2},
$$


where $\mathbf{P}$ is the total momentum, $\mathbf{R}$ is the center-of-mass position, and $T_{\mathrm{r}}$ is the relative kinetic energy, and succeeded in generalizing it in the form of inequalities when the power $q=2$ is replaced by another power $q \geqslant 1$. Then the Hamiltonian (1) or its translation-invariant part (5) with $V \propto r_{i j}^{q}$ is bounded (on both sides) by independent-particle Hamiltonians with a potential proportional to $r_{i}^{q}$. This leads to

$$
\begin{gathered}
E_{N} \geqslant 2^{(q-4) /(q+2)}\left[N^{(2 / q+2)} f_{N}-N^{4-q /(q+2)} E_{2}\right], \\
E_{N} \leqslant 2^{-q /(q+2)}\left[N^{2 /(q+2)} f_{N}-N^{q /(q+2)} E_{2}\right],
\end{gathered}
$$

for $1 \leqslant q \leqslant 2$. The inequalities are reversed for $q \geqslant 2$.

Clearly from Fig. 1, Basdevant and Martin always give the best bound near $q=2$, but their result quickly deteriorates when the potential departs from the harmonic case. Our inequality is saturated at $q=2$ for $N=3$ fermions and for some spin configurations of $N=4$, but saturation is lost at larger $N$. For small systems, our bound dramatically improves that of Lévy-Leblond.

Our result can be extended to more general Hamiltonians, for instance with spin-spin interaction or with external constraints. More details will be given elsewhere. Let us just mention that our bound can be applied to the "quantum dot" systems

$$
H=\sum_{i=1}^{N} \frac{\mathbf{p}_{i}^{2}}{2 m}+\frac{m \omega^{2}}{2} \mathbf{r}_{i}^{2}+\sum_{i<j} \frac{e^{2}}{r_{i j}}
$$

once the center-of-mass contribution (a mere harmonic oscillator) is removed. In the case of $N=3$ electrons with mass $m=1$ and charge $e=1$, one obtains, for $S=3 / 2$ and orbital momentum and parity $L^{P}=1^{+}$, a bound $\epsilon \geqslant 0.1668$ if the oscillator frequency is $\omega=0.01$ and $\epsilon$ $\geqslant 54.968$ if $\omega=10$, to be compared to the exact values $\epsilon=0.1680$ and $\epsilon=54.973$. For $N=4, S=2, L^{P}=0^{-}$, one obtains $\epsilon \geqslant 0.297$ if $\omega=0.01$ and $\epsilon \geqslant 84.895$ if $\omega=10$, close to the exact values $\epsilon=0.299$ and $\epsilon=84.907$. The bound is better for large $\omega$, as the system becomes a pure oscillator.

To conclude, we have derived a lower bound on the energy of $N$-fermion systems, which is independent of the shape of the potential. For small systems, it improves significantly a previous bound expressed in terms of $(N-1)$ independent fermions.

Saturation is not always obtained for the harmonic oscillator. When our bound is iterated to express the $N$-body energy in terms of the two-body energy, the correct large- $N$ behavior is not reached in the case of maximum spin. For other spin configurations, further studies are required.

We would like to thank Ica Stancu, Jean-Louis Basdevant, André Martin, and Piet van Isacker for very useful comments, and Vladimir Belyaev for pointing out useful references. The research of K. V. was sponsored by the U. S. Department of Energy under Contract No. DE-AC0500OR22725 with the Oak Ridge National Laboratory, managed by UT-Battelle, LLC.
${ }^{1}$ M.E. Fisher and D. Ruelle, J. Math. Phys. 7, 260 (1966); F.J. Dyson and A. Lenard, ibid. 8, 423 (1967); J.-M. Lévy-Leblond, ibid. 10, 806 (1969).

${ }^{2}$ R.L. Hall and H.R. Post, Proc. Phys. Soc. London 90, 381 (1967); J.-L. Basdevant, A. Martin, and J.-M. Richard, Nucl. Phys. B 343, 60 (1990).

${ }^{3}$ J.-L. Basdevant, A. Martin, J.-M. Richard, and T.T. Wu, Nucl. Phys. B 393, 111 (1993); A. Benslama, A. Metala, A. Bachkhaznadji, S.R. Zouzou, A. Krikeb, J.-L. Basdevant, J.-M. Richard, and T.T. Wu, Few-Body Syst. 24, 39 (1998).

${ }^{4}$ J.-L. Basdevant and A. Martin, J. Math. Phys. 37, 5916 (1996).

${ }^{5}$ J.-P. Ader, J.-M. Richard, and P. Taxil, Phys. Rev. D 25, 2370 (1982); S. Nussinov, Phys. Rev. Lett. 51, 2081 (1983); J.-M. Richard, Phys. Lett. 139B, 408 (1984).
${ }^{6}$ See, for instance, M. Hamermesh, Group Theory and its Application to Physical Problems, 2nd ed. (Addison-Wesley, Reading, MA, 1964); Fl. Stancu, Group Theory in Subnuclear Physics (Clarendon Press, Oxford, 1996).

${ }^{7}$ See, for instance, S. Fleck and J.-M. Richard, Few-Body Syst. 19, 19 (1995), and references therein.

${ }^{8}$ See, for instance, K. Varga and Y. Suzuki, Phys. Rev. C 52, 2885 (1995); Y. Suzuki and K. Varga, Stochastic Variational Approach to Quantum-Mechanical Few-Body Problems, Lecture Notes in Physics (Springer-Verlag, Berlin, 1998).

${ }^{9}$ R.J.M. Carr and H.R. Post, J. Phys. A 1, 596 (1968); 10, L59 (1977); M.R. Manning, ibid. 5, 855 (1978); E.B. Balbutsev, ibid. 14, 369 (1981); and references therein. 